\title{
EFECTOS DE LA DENSIDAD DE PLANTACIÓN Y LA ALTURA DE PODA SOBRE LA PRODUCCION DE Pinus taeda L. A LOS 5 AÑOS DE EDAD
}

\author{
Ruben Costas ${ }^{1}$ \\ Patricio Mac Donagh \\ Elizabeth Weber \\ Pedro Irschick \\ José Palavecino
}

\begin{abstract}
RESUMEN
En un ensayo con 3 niveles de densidad y 11 niveles de altura de poda, se evaluaron los efectos de la densidad, la altura de poda y la interacción entre ambas sobre la producción de la masa de Pinus taeda L. a los 5 años de edad. Las variables sobre las cuales se analizó la producción fueron el volumen por unidad de superficie, el área basal, el volumen individual, el diámetro a la altura de pecho y la altura.Se observaron mayores volumen por unidad de superficie y área basal y menores volúmenes individuales y los diámetros a la altura de pecho en los tratamientos con mayor densidad. Para todas las variables respuestas, excepto la altura, se observó que en general a mayor altura de poda se produjeron valores medios menores y viceversa.La interacción entre densidad y altura de poda no fue significativa para ninguna de las variables respuestas.

Palabras clave: Densidad; Altura de Poda; Volumen por unidad de superficie; Volumen Individual; Diámetro a la altura de pecho
\end{abstract}

\section{STAND DENSITY AND PRUNE HEIGTH EFFECTS OVER FIVE YEAR OLD YIELD PLANTATION OF Pinus taeda $\mathbf{L}$.}

\begin{abstract}
ABSTRAC
The effects of the stand density and prune height, and the interaction between them, have been evaluated over the yield of five years old Pinus taeda plantation. There were three density levels and eleven prune heights. There were analyzed volume per hectare, basal area, tree volume, diameter at breast height (dbh) and height. It were found higher volume and basal area per hectare, and lower tree volume and dbh for the high density treatments. Except tree height, all the others variables showed lower average values with the increasing of the prune height. The interaction between stand density and prune height was not significant for all analyzed variables.
\end{abstract}

Key-words: Stand density; Prune height; Volume per hectare; Tree volume; Diameter at breast height

\section{INTRODUCCION}

Existe un dilema productivo y económico para el silvicultor respecto de las opciones de manejo de la masa forestal desde el momento de implantación y durante la conducción de la misma.

En un extremo está la maximización de la producción total de madera, la cual se consigue haciendo un uso completo del sitio de implantación, Avery \& Burkhart (1994) a través de:

a) manejo de la masa con regímenes de aclareos sucesivos tales que se interviene cuando los niveles de competencia están al borde de la mortandad de los dominados, y b) Mantenimiento de la mayor cantidad de copa viva por individuo posible, Hawley \& Smith (1972).

En otro extremo está la maximización del diámetro promedio de los árboles de la masa y la obtención de trozas basales libres de nudos, de manera de obtener en la cosecha forestal madera de valor económico unitario alto a través de:

a) manejo de la masa tal que con densidades bajas o intermedias se maximice el espacio disponible para cada individuo de manera que

\footnotetext{
${ }^{1}$ Instituto Subtropical de Investigaciones Forestales, UNaM. Eldorado, Misiones, Argentina. rcostas@facfor.unam.edu.ar
} 
desarrolle su copa sin competencia con los otros individuos de la misma, Daniel et al (1982), y

b) Prácticas de podas sucesivas de manera que al momento de la corta final se obtengan trozas basales donde las marcas o cicatrices de las ramas estén circunscriptas a un cilindro central pequeño, Fassola (1992).

Fahler et al (1986) y Costas et al (2000), a edades de plantación de 8 y 4 años respectivamente, encontraron que menores densidades de plantación inicial produjeron mayores diámetros a la altura de pecho (d.a.p.) medios, y mayores volúmenes por unidad de superficie. Sanquetta et al (2000), mostraron por medio de simulación que espaciamientos iniciales mayores y regímenes de raleos más intensos producen mayor volumen laminable por unidad de superficie al final del turno de rotación que espaciamientos iniciales menores y regímenes de raleos menos intensos. Crechi et al (2001), mostraron que la aplicación de raleos suaves produjeron mayores área basal y volumen total y menor d.a.p. medio que raleos drásticos.

Los objetivos del presente trabajo fueron evaluar los efectos de la densidad, la altura de poda y la interacción entre ambas sobre la producción a los 5 años de edad del volumen total por unidad de superficie, del volumen total individual, del área basal, del diámetro a la altura de pecho y de la altura total.

\section{MATERIALES Y MÉTODOS}

El material experimental es una masa regular de Pinus taeda L. origen Marion, implantada en 1996 en la localidad de Garuhapé-mí, Dto. Gral. Libertador Gral. San Martín, Misiones. Las coordenadas geográficas de ubicación del ensayo son 26 50'00" de latitud Sur y 54²3'30" de longitud Oeste. La superficie total del ensayo es de $19.080 \mathrm{~m}^{2}$. La pendiente promedio del lote es de 3\%. El suelo es homogéneo en todo el ensayo y corresponde a un Kandiudult típico, Soil Taxonomy (1991). El material implantado es homogéneo respecto de la procedencia genética, sitio y tratamientos culturales. El ensayo fue instalado en una plantación cuyo propietario es el Ing. Pedro Irschick. A los 3 años de edad, sobre dos tercios del área del ensayo fueron realizados raleos sistemáticos, producto de los cuales un tercio de la plantación quedó con un promedio de 525 árboles/ha (D1), el otro tercio raleado con un promedio de 760 árboles/ha (D2) y el tercio no raleado quedó con un promedio de 1480 árboles/ha (D3). Ese mismo año fue realizada una poda homogénea en todo el área hasta una altura promedio de $1.80 \mathrm{~m}$.

El ensayo propiamente dicho fue instalado a los 4 años de edad. En cada bloque con una densidad dada fueron asignados al azar los tratamientos de poda descriptos en la tab. 1, los cuales se terminaron de aplicar al sexto año de edad. Las alturas finales de poda adoptadas fueron similares a las practicadas en plantaciones de coníferas en Misiones. Para llegar a cada una de ellas con 2, 3 o 4 levantes de poda fue necesario realizar un levantamiento gradual que se presentan en la tab. 1. Cada uno de los 11 tratamientos diferentes de poda aplicados a los 4 años de edad en cada densidad tuvo por lo menos dos parcelas como repeticiones. La altura total promedio de los árboles al momento de la realización de las podas fue de $7.23 \mathrm{~m}$, sin diferencias significativas de alturas medias entre los sectores con distinta densidad, Costas et al (2001). Las alturas de poda consideradas en el análisis fueron los largos de las varas utilizadas por los operarios que realizaron las podas.

Las superficies de las parcelas utilizadas como unidades experimentales fueron de $252 \mathrm{~m}^{2}$ en D1, $258 \mathrm{~m}^{2}$ en D2 y de $102 \mathrm{~m}^{2}$ en D3.

Cada sector del ensayo con su respectiva densidad fue rodeado de líneas de bordura con densidad igual a cada sector, sobre las cuales no se realizó ninguna medición y tienen la función de evitar la influencia de un nivel de densidad sobre otro. 
Efectos de la densidad de plantación...

Tabla 1: Esquema general de tratamientos aplicados en el ensayo

Table 1: General lay out of applied treatments in the trial

\begin{tabular}{|c|c|c|c|c|c|c|}
\hline DENS & $\begin{array}{c}\text { ALTURA } \\
\text { FINAL } \\
\text { DE PODA (m) }\end{array}$ & $\begin{array}{c}\mathrm{N}^{\circ} \mathrm{DE} \\
\mathrm{LEVANT} \\
\mathrm{ES} \\
\mathrm{DE} \\
\text { PODA }\end{array}$ & $\begin{array}{c}\text { ALTURA } \\
\text { DE PODA } \\
\text { ALCANZADA } \\
\text { AÑO } 4(\mathrm{~m})\end{array}$ & $\begin{array}{c}\text { ALTURA } \\
\text { DE PODA } \\
\text { ALCANZADA } \\
\text { AÑO } 5(\mathrm{~m})\end{array}$ & $\begin{array}{c}\text { ALTURA } \\
\text { DE PODA } \\
\text { ALCANZADA } \\
\text { AÑO } 6(\mathrm{~m})\end{array}$ & COMB \\
\hline \multirow{12}{*}{ D1 } & \multirow{3}{*}{$\mathrm{HP} 1=3.30$} & $2 \mathrm{~L}$ & 3.30 & 3.30 & 3.30 & 1.1 \\
\hline & & $3 \mathrm{~L}$ & 2.60 & 3.30 & 3.30 & 1.2 \\
\hline & & $4 \mathrm{~L}$ & 2.37 & 2.83 & 3.30 & 1.3 \\
\hline & \multirow{3}{*}{$\mathrm{HP} 2=4.40$} & $2 \mathrm{~L}$ & 4.40 & 4.40 & 4.40 & 1.4 \\
\hline & & $3 \mathrm{~L}$ & 3.15 & 4.40 & 4.40 & 1.5 \\
\hline & & $4 \mathrm{~L}$ & 2.73 & 3.56 & 4.40 & 1.6 \\
\hline & \multirow{3}{*}{$\mathrm{HP} 3=5.50$} & $2 \mathrm{~L}$ & 1.80 & 5.50 & 5.50 & 1.7 \\
\hline & & $3 \mathrm{~L}$ & 3.70 & 3.70 & 5.50 & 1.8 \\
\hline & & $4 \mathrm{~L}$ & 3.10 & 4.30 & 5.50 & 1.9 \\
\hline & \multirow{3}{*}{$\mathrm{HP} 4=6.60$} & $2 \mathrm{~L}$ & 1.80 & 6.60 & 6.60 & 1.10 \\
\hline & & $3 \mathrm{~L}$ & 4.25 & 4.25 & 6.60 & 1.11 \\
\hline & & $4 \mathrm{~L}$ & 3.47 & 5.03 & 6.60 & 1.12 \\
\hline \multirow{12}{*}{ D2 } & \multirow{3}{*}{$\mathrm{HP} 1=3.30$} & $2 \mathrm{~L}$ & 3.30 & 3.30 & 3.30 & 2.1 \\
\hline & & $3 \mathrm{~L}$ & 2.60 & 3.30 & 3.30 & 2.2 \\
\hline & & $4 \mathrm{~L}$ & 2.37 & 2.83 & 3.30 & 2.3 \\
\hline & \multirow{3}{*}{$\mathrm{HP} 2=4.40$} & $2 \mathrm{~L}$ & 4.40 & 4.40 & 4.40 & 2.4 \\
\hline & & $3 \mathrm{~L}$ & 3.15 & 4.40 & 4.40 & 2.5 \\
\hline & & $4 \mathrm{~L}$ & 2.73 & 3.56 & 4.40 & 2.6 \\
\hline & \multirow{3}{*}{$\mathrm{HP} 3=5.50$} & $2 \mathrm{~L}$ & 1.80 & 5.50 & 5.50 & 2.7 \\
\hline & & $3 \mathrm{~L}$ & 3.70 & 3.70 & 5.50 & 2.8 \\
\hline & & $4 \mathrm{~L}$ & 3.10 & 4.30 & 5.50 & 2.9 \\
\hline & \multirow{3}{*}{$\mathrm{HP} 4=6.60$} & $2 \mathrm{~L}$ & 1.80 & 6.60 & 6.60 & 2.10 \\
\hline & & $3 \mathrm{~L}$ & 4.25 & 4.25 & 6.60 & 2.11 \\
\hline & & $4 \mathrm{~L}$ & 3.47 & 5.03 & 6.60 & 2.12 \\
\hline \multirow{12}{*}{ D3 } & \multirow{3}{*}{$\mathrm{HP} 1=3.30$} & $2 \mathrm{~L}$ & 3.30 & 3.30 & 3.30 & 3.1 \\
\hline & & $3 \mathrm{~L}$ & 2.60 & 3.30 & 3.30 & 3.2 \\
\hline & & $4 \mathrm{~L}$ & 2.37 & 2.83 & 3.30 & 3.3 \\
\hline & \multirow{3}{*}{$\mathrm{HP} 2=4.40$} & $2 \mathrm{~L}$ & 4.40 & 4.40 & 4.40 & 3.4 \\
\hline & & $3 \mathrm{~L}$ & 3.15 & 4.40 & 4.40 & 3.5 \\
\hline & & $4 \mathrm{~L}$ & 2.73 & 3.56 & 4.40 & 3.6 \\
\hline & \multirow{3}{*}{$\mathrm{HP} 3=5.50$} & $2 \mathrm{~L}$ & 1.80 & 5.50 & 5.50 & 3.7 \\
\hline & & $3 \mathrm{~L}$ & 3.70 & 3.70 & 5.50 & 3.8 \\
\hline & & $4 \mathrm{~L}$ & 3.10 & 4.30 & 5.50 & 3.9 \\
\hline & \multirow{3}{*}{$\mathrm{HP} 4=6.60$} & $2 \mathrm{~L}$ & 1.80 & 6.60 & 6.60 & 3.10 \\
\hline & & $3 \mathrm{~L}$ & 4.25 & 4.25 & 6.60 & 3.11 \\
\hline & & $4 \mathrm{~L}$ & 3.47 & 5.03 & 6.60 & 3.12 \\
\hline
\end{tabular}


A los 5 años de edad, se analizaron los efectos de la densidad, de las podas efectuadas en el año 4 y la interacción entre ellas sobre las cinco variables respuestas enumeradas a continuación:

a) Volumen individual promedio $\left(\mathrm{dm}^{3}\right)$. Fue calculado primero para cada unidad experimental a partir de los volúmenes individuales predichos por la siguiente función de volumen;

$$
\begin{gathered}
\mathrm{V}=0.06081)^{*}\left(\mathrm{~d}^{\wedge} 1.89376\right) *\left(\mathrm{~h}^{\wedge} 0.92716\right) *(1 \\
\left.-\left(96.363^{*}\left(\mathrm{~d}^{\wedge}(-0.565)\right) / 100\right)\right)
\end{gathered}
$$

Donde $\mathrm{V}$ es el volumen total $\left(\mathrm{dm}^{3}\right), \mathrm{d}$ es el diámetro a la altura de pecho $(\mathrm{cm})$ y $\mathrm{h}$ es la altura total del árbol (m).

b) Volumen total por unidad de superficie (m3/ha);

c) Diámetro a la altura de pecho promedio (cm);

d) Altura promedio (m);

e) Area Basal $\left(\mathrm{m}^{2} / \mathrm{ha}\right)$.

En todos los análisis se incluyeron como covariables los valores que cada una de las variables analizadas tuvieron antes de la aplicación de los tratamientos de poda, con el fin de poder explicar las respuestas a los tratamientos teniendo en cuenta los valores iniciales, aumentando la sensibilidad de las pruebas de comparaciones múltiples.

La comprobación del cumplimiento de los supuestos mostró leves apartamientos de la distribución normal de los residuales y de la homogeneidad de variancia para las cinco variables respuestas. Las transformaciones $\log (\mathrm{y}), \ln (\mathrm{y})$ e $\mathrm{y} \lambda$ donde $\lambda=1-\alpha$, siendo $\alpha$ obtenido por regresión lineal simple de $\log \mathrm{Si}$ $=\log \theta+\alpha \log 8 \mathrm{i}$ no produjeron mejoras importantes de la distribución de los residuos. Dado que las pruebas realizadas con modelos de efectos fijos como los aplicados en este ensayo son robustas frente a apartamientos leves de los supuestos, Montgomery (1991), se optó por presentar los resultados del análisis con las observaciones sin transformar.

\section{RESULTADOS Y DISCUSIÓN}

\section{Variable repuesta: Volumen individual promedio (VIND5)}

Analizando los resultados mostrados en la tab. 2, se observa que hubo diferencias de VIND5 entre las densidades. Los resultados de la prueba de Duncan indicaron que las densidades D1 y D2 resultaron homogéneas entre sí y superiores a la densidad D3 respecto de los VIND5. De los resultados se desprende que ya a la edad de 5 años las densidades más bajas producen volúmenes individuales promedios mayores.

Aunque no se manifestó una clara asociación entre los VIND5 y las alturas de poda efectuada, se detectaron diferencias significativas entre los VIND5 promedios producidos en cada nivel de HP. Los resultados del test de Duncan para la diferencia de medias mostrados en la tab. 2, que los 3 niveles de menor HP produjeron resultados significativamente superiores que el resto de los tratamientos. La incorporación en el análisis de la covariable volumen individual promedio producido a los 4 años de edad (VIND4) en cada tratamiento sirve para explicar parte de las respuestas de VIND5 a las distintas alturas de poda. Por ejemplo el VIND4 medio del tratamiento de poda hasta una altura de $2.73 \mathrm{~m}$ era uno de los dos menores antes de la aplicación de las podas, con lo cual es probable que el VIND5 producido esté más afectado por el valor inicial que por el efecto de la práctica de poda.

\section{Variable repuesta: Volumen por unidad de superficie (VHA5)}

Se encontraron diferencias

significativas entre las medias de VHA5 debidas al efecto de la densidad. A su vez, según la prueba de Duncan se observó que la media de VHA5 de la densidad D3 fue significativamente superior a la producida en la densidad D2 y ésta a su vez mayor a la producida en la densidad D1.

Sin manifestarse claramente menores valores de VHA5 a alturas de poda mayores, hubo diferencias significativas entre las medias de VHA5 debidas al efecto de la altura de poda. A través de a prueba de Duncan se observó que las medias de VHA5 producidas 
en 2 de los 3 niveles de menor HP (2.37 $\mathrm{m} \mathrm{y}$ $2.60 \mathrm{~m}$ ), fueron significativamente superiores a las dadas en 2 de los 3 niveles de mayor HP (4.25 m y $3.70 \mathrm{~m})$.

\section{Variable respuesta: Diámetro a la altura de pecho (DAP5).}

Las medias de DAP5 producidas en las dos densidades menores resultaron estadísticamente homogéneas entre sí y superiores al DAP5 de la densidad mayor.

El efecto de la altura de levante de poda fue significativo respecto del DAP5. Según la prueba de Duncan, las medias DAP5 producidas en los 3 niveles de menor HP resultaron ser estadísticamente superiores a los 3 niveles de mayor HP.

Los resultados del test de Duncan para la variable DAP5 son similares a los obtenidos para la variable VIND5. Los valores de la covariable DAP4 permiten explicar parte de los resultados obtenidos de VIND5.

\section{Variable respuesta: Altura media (ALT5).}

Según las pruebas $\mathrm{F}$ y de Tukey, los promedios de ALT5 en cada nivel de densidad resultaron ser estadísticamente homogéneos entre sí. La prueba de Duncan, más sensible que la prueba de Tukey, indicó que en la mayor densidad se produjo una altura media superior a las otras dos densidades. Los resultados son mostrados en la tab. 5 .

Si bien según las pruebas $\mathrm{F}$, de Tukey y Duncan hubo diferencias significativas entre las medias de ALT5 producidas en cada nivel de HP, no se pudo encontrar ninguna asociación entre la altura de poda y ALT5. Es probable que los resultados estén mucho más influenciados por los valores iniciales y por variación no controlada que por los tratamientos de poda.

\section{Variable respuesta: Area Basal (AB5).}

La densidad produjo efectos significativos sobre el AB5, siendo la media en la mayor densidad significativamente superior al AB5 de la densidad intermedia y ésta a su vez superior a la producida en la densidad menor.

Se observaron diferencias significativas de AB5 debidas al efecto de la altura de poda, tal cual se muestra en la tab. 6 . Si bien no hay una clara asociación entre el AB5 y la altura de poda, según la prueba de Duncan los 3 niveles de menor altura de poda produjeron $\mathrm{AB} 5$ mayores que las resultantes en los 3 niveles de mayor altura de poda. Los resultados se muestran en la tab. 6 .

Tabla 2: Resultados de la prueba F, VIND5 promedios en cada densidad y en cada nivel de poda e identificación de cada tratamiento según Duncan

Table 2: Results of F tests, means of VIND5 in each density and prune level and Duncan identification for each treatment

\begin{tabular}{|c|c|c|c|c|c|}
\hline \multicolumn{3}{|c|}{ EFECTOS DE DENSIDAD } & \multicolumn{3}{|c|}{ EFECTOS DE LA PODA } \\
\hline \multicolumn{3}{|c|}{$\mathrm{F}(2,38)=.09 ; \mathrm{p}<.9155$} & \multicolumn{3}{|c|}{$\mathrm{F}(10,38)=4.25 ; \mathrm{p}<.0005$} \\
\hline DENS(arb/ha) & VIND5 $\left(\mathrm{dm}^{3}\right)$ & VIND4 $\left(\mathrm{dm}^{3}\right)$ & $\mathrm{HP}(\mathrm{m})$ & VIND5 $\left(\mathrm{dm}^{3}\right)$ & VIND4 $\left(\mathrm{dm}^{3}\right)$ \\
\hline 525 & $64,23^{\mathrm{a}}$ & 30.51 & 2,60 & $72,13^{\mathrm{a}}$ & 30.30 \\
\hline 760 & $62,40^{\mathrm{a}}$ & 29.73 & 2,37 & $69,58^{\mathrm{a}}$ & 30.31 \\
\hline 1480 & $51,82^{b}$ & 22.38 & 1,80 & $67.97^{\mathrm{a}}$ & 30.61 \\
\hline \multirow{8}{*}{\multicolumn{3}{|c|}{$\begin{array}{l}\text { La interacción entre los efectos de la densidad y } \\
\text { de la altura de poda no fue significativa sobre VIND5 } \\
\text { con lo cual se puede concluir que cada uno de los } \\
\text { factores actúa independientemente sobre el VIND5 }\end{array}$}} & 3,47 & $60.60^{b}$ & 28.92 \\
\hline & & & 3,15 & $59.30^{\mathrm{b}}$ & 27.12 \\
\hline & & & 3,10 & $58.68^{\mathrm{b}}$ & 28.24 \\
\hline & & & 4,40 & $57.17^{\mathrm{b}}$ & 29.16 \\
\hline & & & 3,30 & $55.51^{\mathrm{bc}}$ & 26.99 \\
\hline & & & 4,25 & $50.17^{\mathrm{c}}$ & 27.29 \\
\hline & & & 2,73 & $47.48^{\mathrm{c}}$ & 22.20 \\
\hline & & & 3,70 & $47.25^{\mathrm{c}}$ & 21.80 \\
\hline
\end{tabular}

$(*)$ Tratamientos identificados con letras iguales indican que entre ellos no hay diferencias significativas con un nivel de confianza del $95 \%$. 
Tabla 3: Resultados de la prueba F, Medias de VHA5 en cada nivel de densidad y altura de poda e identificación de cada tratamiento según Duncan

Table 3: $\quad$ Results of F tests, means of VHA5 in each density and prune level and Duncan identification for each treatment

\begin{tabular}{|c|c|c|c|c|c|}
\hline \multicolumn{3}{|c|}{ EFECTOS DE DENSIDAD } & \multicolumn{3}{|c|}{ EFECTOS DE LA PODA } \\
\hline \multicolumn{3}{|c|}{$\mathrm{F}(2,38)=10.97 ; \mathrm{p}<.0002$} & \multicolumn{3}{|c|}{$\mathrm{F}(10,38)=5.02 ; \mathrm{p}<.0001$} \\
\hline $\operatorname{VHA} 5\left(\mathrm{~m}^{3} / \mathrm{ha}\right)$ & VHA5 $\left(\mathrm{m}^{3} / \mathrm{ha}\right)$ & VHA4 $\left(\mathrm{m}^{3} / \mathrm{ha}\right)$ & $\mathrm{HP}(\mathrm{m})$ & $\operatorname{VHA} 5\left(\mathrm{~m}^{3} / \mathrm{ha}\right)$ & VHA4(m $\left.{ }^{3} / \mathrm{ha}\right)$ \\
\hline 1480 & $76.91^{\mathrm{a}}$ & 35.46 & 2.37 & $66.02^{a}$ & 27.63 \\
\hline 760 & $47.52^{b}$ & 22.02 & 2.60 & $61.74^{\text {ab }}$ & 26.03 \\
\hline 525 & $33.83^{\mathrm{c}}$ & 15.23 & 3.47 & $59.60^{\mathrm{ab}}$ & 28.29 \\
\hline \multirow{8}{*}{\multicolumn{3}{|c|}{$\begin{array}{l}\text { La interacción entre la densidad y la altura de poda no } \\
\text { fue significativa respecto de VHA5. }\end{array}$}} & 1.80 & $57.64^{\mathrm{b}}$ & 24.19 \\
\hline & & & 3.15 & $55.40^{\mathrm{bc}}$ & 25.73 \\
\hline & & & 3.30 & $53.53^{\mathrm{bc}}$ & 27.16 \\
\hline & & & 4.40 & $53.46^{\mathrm{bc}}$ & 26.27 \\
\hline & & & 3.10 & $49.44^{\mathrm{c}}$ & 23.22 \\
\hline & & & 4.25 & $40.93^{\mathrm{d}}$ & 21.80 \\
\hline & & & 2.73 & $40.23^{\mathrm{d}}$ & 18.41 \\
\hline & & & 3.70 & $37.42^{\mathrm{d}}$ & 17.90 \\
\hline
\end{tabular}

Tabla 4: Resultados de la prueba F, Medias de DAP5 en cada nivel de densidad y altura de poda e identificación de cada tratamiento según Duncan

Table 4: $\quad$ Results of F tests, means of DAP5 in each density and prune level and Duncan identification for each treatment

\begin{tabular}{|c|c|c|c|c|c|}
\hline \multicolumn{3}{|c|}{ EFECTOS DE DENSIDAD } & \multicolumn{3}{|c|}{ EFECTOS DE LA PODA } \\
\hline \multicolumn{3}{|c|}{$\mathrm{F}(2,38)=2.45 ; \mathrm{p}<.1001$} & \multicolumn{3}{|c|}{$\mathrm{F}(10,38)=4.65 ; \mathrm{p}<.0002$} \\
\hline DENS(arb/ha) & DAP5 $(\mathrm{cm})$ & DAP4 $(\mathrm{cm})$ & $\mathrm{HP}(\mathrm{m})$ & DAP5 $(\mathrm{cm})$ & DAP4 $(\mathrm{cm})$ \\
\hline 525 & $14,45^{\mathrm{a}}$ & 11.54 & 2.60 & $15.02^{\mathrm{a}}$ & 11.47 \\
\hline 760 & $14,30^{\mathrm{a}}$ & 11.51 & 1.80 & $14.88^{\mathrm{a}}$ & 11.58 \\
\hline 1480 & $12,76^{\mathrm{b}}$ & 10.00 & 2.37 & $14.82^{\mathrm{ab}}$ & 11.22 \\
\hline \multirow{8}{*}{\multicolumn{3}{|c|}{$\begin{array}{l}\text { La interacción entre la densidad y la altura de poda no } \\
\text { fue significativa respecto del DAP5. }\end{array}$}} & 3.47 & $14.07^{\mathrm{bc}}$ & 11.32 \\
\hline & & & 3.15 & $14.04^{\mathrm{bc}}$ & 11.02 \\
\hline & & & 3.10 & $13.90^{\mathrm{c}}$ & 11.27 \\
\hline & & & 3.30 & $13.69^{\mathrm{cd}}$ & 10.89 \\
\hline & & & 4.40 & $13.39^{\mathrm{cd}}$ & 11.29 \\
\hline & & & 4.25 & $12.95^{\mathrm{de}}$ & 10.98 \\
\hline & & & 2.73 & $12.89^{\mathrm{de}}$ & 10.03 \\
\hline & & & 3.70 & $12.58^{\mathrm{e}}$ & 10.11 \\
\hline
\end{tabular}

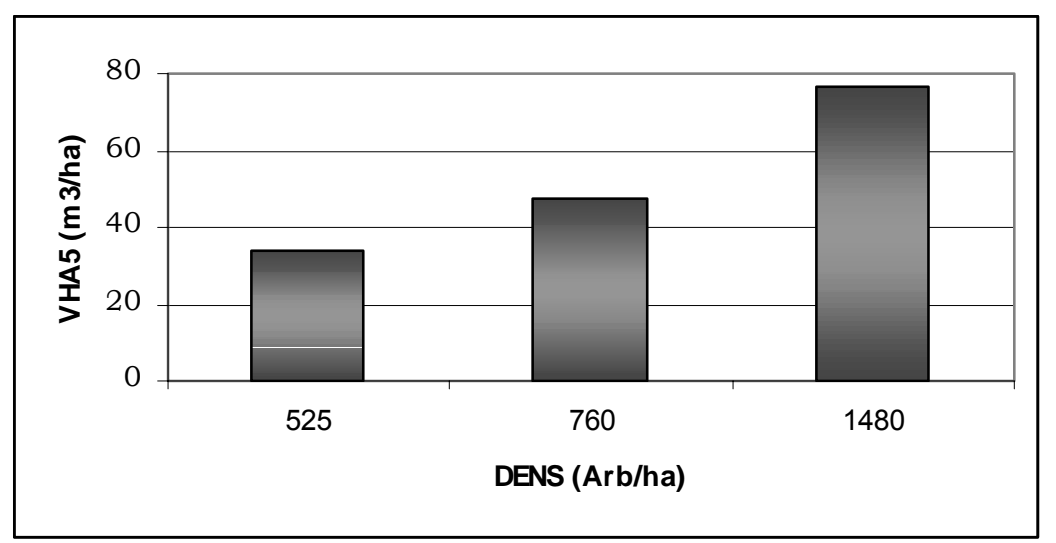

Figura 1: Medias de VHA5 $\left(\mathrm{m}^{3} / \mathrm{ha}\right)$ de cada densidad Figure 1: Means of VHA5 in each density level 


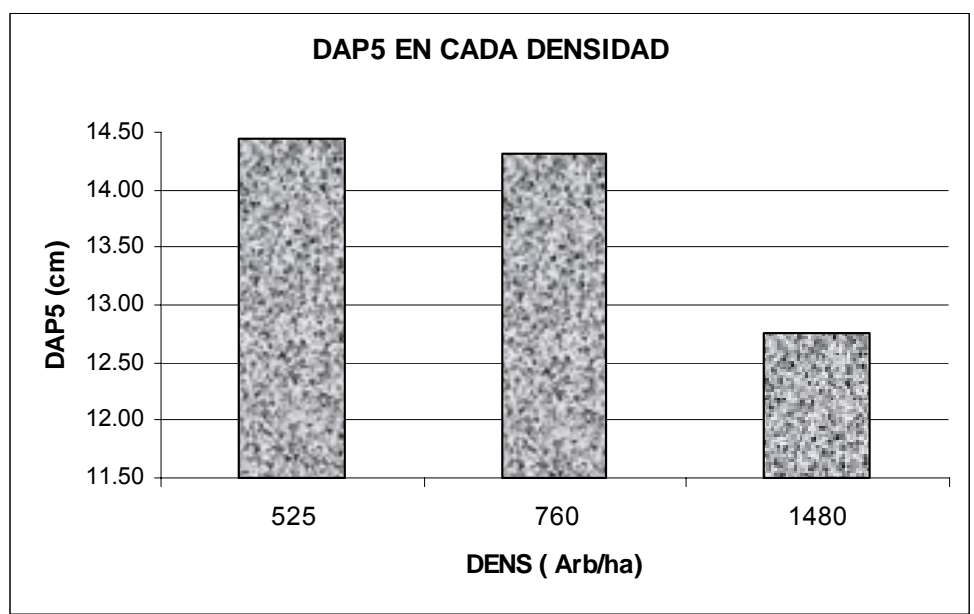

Figura 2: Medias de DAP5 $(\mathrm{cm})$ de cada densidad

Figure 2: Means of DAP5 $(\mathrm{cm})$ in each density level

Tabla 5: Resultados de la prueba F, Medias de ALT5 en cada nivel de densidad y altura de poda e identificación de cada tratamiento según Duncan

Table 5: $\quad$ Results of F tests, means of ALT5 in each density and prune level and Duncan identification for each treatment

\begin{tabular}{|c|c|c|c|c|c|}
\hline \multicolumn{3}{|c|}{ EFECTOS DE DENSIDAD } & \multicolumn{3}{|c|}{ EFECTOS DE LA PODA } \\
\hline \multicolumn{3}{|c|}{$\mathrm{F}(2,38)=3.04 ; \mathrm{p}<.0595$} & \multicolumn{3}{|c|}{$\mathrm{F}(10,38)=2.13 ; \mathrm{p}<.0462$} \\
\hline DENS(arb/ha) & ALT5(m) & ALT4(m) & $\mathrm{HP}(\mathrm{m})$ & ALT5 (m) & ALT4(m) \\
\hline 1480 & $9,51^{\mathrm{a}}$ & 7.22 & 2.60 & $9.89^{\mathrm{a}}$ & 7.34 \\
\hline 760 & $9,18^{b}$ & 7.23 & 4.40 & $9.80^{\mathrm{a}}$ & 7.35 \\
\hline 525 & $9,17^{b}$ & 7.23 & 2.37 & $9.54^{\mathrm{ab}}$ & 7.33 \\
\hline \multirow{8}{*}{\multicolumn{3}{|c|}{$\begin{array}{l}\text { La interacción entre la densidad y la altura de poda no fue } \\
\text { significativa respecto de la ALT5. }\end{array}$}} & 3.47 & $9.52^{\mathrm{ab}}$ & 7.34 \\
\hline & & & 1.80 & $9.35^{\mathrm{abc}}$ & 7.23 \\
\hline & & & 3.30 & $9.25^{a b c}$ & 7.39 \\
\hline & & & 3.15 & $9.22^{a b c}$ & 7.21 \\
\hline & & & 3.10 & $9.15^{\mathrm{abc}}$ & 7.11 \\
\hline & & & 4.25 & $8.96^{\mathrm{bc}}$ & 7.24 \\
\hline & & & 3.70 & $8.75^{c}$ & 6.69 \\
\hline & & & 2.73 & $8.70^{\mathrm{c}}$ & 7.28 \\
\hline
\end{tabular}

Tabla 6: Resultados de la prueba F, Medias de AB5 en cada nivel de densidad y altura de poda e identificación de tratamientos según Duncan

Table 6: Results of F tests, means of VIND5 in each density and prune level and Duncan identification for each treatment

\begin{tabular}{|c|c|c|c|c|c|}
\hline \multicolumn{3}{|c|}{ EFECTOS DE DENSIDAD } & \multicolumn{3}{|c|}{ EFECTOS DE LA PODA } \\
\hline \multicolumn{3}{|c|}{$\mathrm{F}(2,38)=7.80 ; \mathrm{p}<.0015$} & \multicolumn{3}{|c|}{$\mathrm{F}(10,38)=6.99 ; \mathrm{p}<.0000$} \\
\hline DENS (arb/ha) & $\begin{array}{c}\mathrm{AB} 5 \\
\left(\mathrm{~m}^{2} / \mathrm{ha}\right)\end{array}$ & $\begin{array}{c}\mathrm{AB} 4 \\
\left(\mathrm{~m}^{2} / \mathrm{ha}\right)\end{array}$ & $\begin{array}{l}\text { PODA } \\
(\mathrm{m})\end{array}$ & $\begin{array}{c}\mathrm{AB} 5 \\
\left(\mathrm{~m}^{2} / \mathrm{ha}\right)\end{array}$ & $\begin{array}{c}\mathrm{AB} 4 \\
\left(\mathrm{~m}^{2} / \mathrm{ha}\right)\end{array}$ \\
\hline 1480 & $20,07^{a}$ & 12.35 & 2.37 & $17,07^{\mathrm{a}}$ & 9.41 \\
\hline 760 & $12,88^{b}$ & 7.72 & 2.60 & $15,77^{b}$ & 9.08 \\
\hline 525 & $9,14^{\mathrm{c}}$ & 5.35 & 3.47 & $15,61^{b c}$ & 9.73 \\
\hline \multirow{8}{*}{\multicolumn{3}{|c|}{$\begin{array}{l}\text { La interacción entre la densidad y la altura de poda no } \\
\text { fue significativa respecto de la AB5. }\end{array}$}} & 1.80 & $15,26^{\mathrm{bc}}$ & 8.59 \\
\hline & & & 3.15 & $15,01^{b c}$ & 9.09 \\
\hline & & & 3.30 & $14,38^{\mathrm{cd}}$ & 9.14 \\
\hline & & & 4.40 & $13,63^{\mathrm{d}}$ & 9.23 \\
\hline & & & 3.10 & $13,32^{\mathrm{d}}$ & 8.23 \\
\hline & & & 2.73 & $11,43^{\mathrm{e}}$ & 6.48 \\
\hline & & & 4.25 & $11,11^{\mathrm{e}}$ & 7.51 \\
\hline & & & 3.70 & $10,51^{\mathrm{e}}$ & 6.70 \\
\hline
\end{tabular}




\section{DISCUSIÓN Y CONCLUSIONES}

Los resultados de los análisis del efecto de la densidad sobre las 5 variables bajo estudio, fueron similares a los obtenidos por Fahler et al (1986) a los 8 años de edad y Crechi et al (2001) a los 13 años de edad. En el caso del presente trabajo los resultados obtenidos fueron producidos a los 5 años de edad.

El efecto de la densidad fue importante para explicar la variación de 4 de las 5 variables respuesta. Respecto de las dos variables que resultan de la agregación de los individuos de la masa, VHA5 y AB5, sus valores medios fueron mayores en los niveles de mayor densidad y viceversa. Ocurrió lo contrario para las variables respuesta que promedian las dimensiones de los individuos de la masa, VIND5 Y DAP5. No se encontró influencia significativa de la densidad sobre la ALT5.

Transcurrido solo un año desde la aplicación de los tratamientos, la incidencia de la altura de poda no resultó clara para explicar la variación de todas las variables respuesta. Sus efectos quedaron confundidos con la influencia de los valores iniciales utilizados como covariables. Es probable que en años posteriores y finalizada la aplicación de los tratamientos de poda previstos en el ensayo, sus efectos sean más marcados que los observados al año siguiente del inicio de la aplicación de tratamientos de podas con distintas alturas.

Respecto de las dos variables que resultan de la agregación de los individuos de la masa, VHA5 y AB5, si bien no se observó una clara disminución de las mismas en los niveles de mayor altura de poda, se observó que el VHA5 y el AB5 de dos de los tres tratamientos con menores alturas de poda fueron significativamente mayores que los producidos en dos de los tres tratamientos de alturas de poda mayores.

Respecto de VIND5 y DAP5, que resultan del promedio de los individuos de la masa, se observó que los valores medios en los 3 tratamientos con menor altura de poda fueron significativamente mayores que los producidos en los 3 tratamientos con mayor altura de poda.

Para las cinco variables respuestas se observó que la interacción entre densidad y altura de poda no fue significativa. Quiere decir que estos dos factores se comportaron en forma aditiva sobre todas las variables analizadas, o sea que la densidad y la altura de poda produjeron efectos independientemente uno del otro.

\section{AGRADECIMIENTOS}

A los alumnos de Ingeniería Forestal de la F.C.F. Rosa A. Winck, Liliana E. Rivero, Víctor Dávalos y Patricia Stopp, por su participación ad-honorem en la toma de datos y parcialmente en el procesamiento de los mismos.

\section{REFERENCIAS}

AVERY T. E. \& BURKHART H. E. (1994) Forest measurements. Fourth Edition. New York: McGraw-Hill.

COSTAS, R., MAC DONAGH P., WEBER, E., IRSCHICK, P. Y PALAVECINO, J. (2001) Pinus taeda L. Densidad de Plantación y Rendimiento. Revista de la Facultad de Ciencias Agrarias - Universidad Nacional de Cuyo. Tomo XXXIIl No 2, p. 11-19

CRECHI, E. H., FRIEDL, R. A., FASSOLA. H. E., FERNANDEZ R. A. Y FAHLER, J. C. Efectos de la Intensidad y Oportunidad de Raleo en Pinus taeda L. sobre el crecimiento y la producción en el noroeste de Misiones, Argentina. $2^{\circ}$ Simpósio Latino-Americano sobre Manejo Florestal. Santa María - RS Brasil. 13 e 14 de setembro de 2001. p 189210.

DANIEL, T. W., HELM, J. A., BACKER, F. S. (1982) Princípios de Silvicultura. Segunda Edición en Inglés. Primera Edición en Castellano. México: Libros McGraw-Hill de México.

FAHLER, J. C., DI LUCCA, C. M., OROZCO E. G., RODRIGUEZ, G. H. Y GIMENEZ, S. (1986). Comportamiento de 10 diferentes densidades de plantación inicial en P. Taeda a los 8 años de edad en suelos del complejo 9 (C.A.R.T.A.) en la provincia de Misiones: E. E. A. Misiones. INTA. Informe Técnico $\mathrm{N}^{\circ}$ 46.

FASSOLA, H. E. (1992) Regímenes silvícolas con producción de madera libre de nudos en $P$. Elliottii Engelm en la zona central de la provincia de Corrientes. Eldorado, Misiones: 
Revista Yvyraretá $N^{\circ} 3$ - F.C.F. - U.Na.M.. p. $28-62$.

HAWLEY R. C., SMITH, D. M., (1972). Silvicultura Práctica. Barcelona: Ediciones Omega S.A.

MONTGOMERY, D.C. (1991) Design and Analysis of experiments. New York: John Wiley \& Sons.

SANQUETTA, C. R.; REZENDE A. V.; GAIAD, D.; SCHAAF, L. B. Y ZAMPIER, A. C. (2000) Produção de madeira para laminação em povoamentos de Pinus taeda submetidos a diferentes densidades e regimes de desbaste: um abordagem experimental. Revista Floresta - Fundação de Pesquisas Florestais do Paraná Volume 28 p. 83-97.

SOIL TAXONOMY. (1991). Key to SOIL TAXONOMY. Soil survey staff. AID. USDA. 